\title{
Humanização no cuidado em saúde mental: compreensões dos enfermeiros
}

\author{
Deivson Wendell da Costa Lima ${ }^{1}$ \\ (1D) http:///orcid.org/0000-0002-7020-2172 \\ Ana Karla Ramalho Paixão \\ (D) http://orcid.org/0000-0003-3900-5549 \\ Kelianny Pinheiro Bezerra ${ }^{2}$ \\ (D) http://orcid.org/0000-0001-9418-5314 \\ Rodrigo Jácob Moreira de Freitas ${ }^{3}$ \\ (D) https://orcid.org/0000-0002-5528-2995 \\ Lívia Dayane Sousa Azevedo 4 \\ (D) https://orcid.org/0000-0002-0019-9342 \\ Fátima Raquel Rosado Morais ${ }^{2}$ \\ (D) https://orcid.org/0000-0001-8052-4725
}

${ }^{1}$ Universidade de São Paulo, Departamento de Enfermagem Psiquiátrica, Ribeirão Preto, SP, Brasil.

${ }^{2}$ Universidade do Estado do Rio Grande do Norte, Faculdade de Enfermagem, Mossoró, RN, Brasil.

${ }^{3}$ Universidade do Estado do Rio Grande do Norte, Departamento de Enfermagem, Pau dos Ferros, RN, Brasil.

${ }^{4}$ Universidade de São Paulo, Faculdade de Medicina, Ribeirão Preto, SP, Brasil.
Objetivo: conhecer as compreensões dos enfermeiros sobre humanização no cuidado em saúde mental. Método: tratase de uma pesquisa exploratória, de abordagem qualitativa, realizada com 12 enfermeiros em um hospital psiquiátrico do interior do Nordeste, Brasil, no período de setembro de 2014 a março de 2015. Para a coleta de dados, utilizou-se entrevista semiestruturada, observação não participante e observação dos registros de Enfermagem, analisando-os a partir da Análise de Conteúdo de Bardin. Resultados: emergiram quatro categorias: acolhimento, autonomia, protagonismo e corresponsabilidade. O cuidado humanizado aparece atrelado ao modelo manicomial, culminando em práticas focadas no uso da medicação, ações desarticuladas e sem participação do paciente no tratamento. A percepção da humanização é de dificuldade de atenção às pessoas em crises psíquicas, o que inviabiliza a produção do cuidado integral. Conclusão: o estudo contribui para a reflexão do cuidado de Enfermagem em saúde mental onde é preciso modificar as relações que o discurso biomédico mantém com os que buscam uma prática humanizada.

Descritores: Enfermagem Psiquiátrica; Cuidados de Enfermagem; Saúde Mental; Humanização da Assistência.

\section{Como citar este artigo}

Lima DWC, Paixão AKR, Bezerra KP, Freitas RJM, Azevedo LDS, Morais FRR. Humanization in mental health care: nurses' understandings. SMAD, Rev Eletrônica Saúde Mental Álcool Drog. 2021 jan.-mar.;17(1):58-65. doi: https://dx.doi.org/10.11606/issn.1806-6976.smad.2021.164401 


\section{Humanization in mental health care: nurses' understandings}

Objective: to know nurses' understandings of humanization in mental health care. Method: it is an exploratory research, with a qualitative approach, carried out with 12 nurses in a psychiatric hospital in the interior of the Northeast, Brazil, from September 2014 to March 2015. For data collection, semi-structured interviews were used, non-participant observation and observation of Nursing records, analyzing them from Bardin's Content Analysis. Results: four categories emerged: welcoming, autonomy, protagonism and co-responsibility. Humanized care appears linked to the asylum model, culminating in practices focused on the use of medication, disjointed actions and without patient participation in the treatment. The perception of humanization is of difficulty in caring for people in psychic crises, which makes the production of comprehensive care unfeasible. Conclusion: the study contributes to the reflection of nursing care in mental health where it is necessary to modify the relationships that biomedical discourse maintains with those who seek a humanized practice.

Descriptors: Psychiatric Nursing; Nursing Care; Mental Health; Humanization of Assistance.

\section{Humanización en la atención de la salud mental: comprensión de las enfermeras}

Objetivo: se objetivó conocer las comprensiones de los enfermeros sobre humanización en el cuidado en salud mental. Método: se trata de una investigación exploratoria de abordaje cualitativo realizada con 12 enfermeros en un hospital psiquiátrico del interior del Nordeste, Brasil, en el período de septiembre de 2014 a marzo de 2015. Para la recolección de datos se utilizó entrevista semiestructurada, observación no participante y observación de los registros de enfermería, analizados a partir del análisis de contenido de Bardin. Resultados: se plantearon cuatro categorías: acogida, autonomía, protagonismo y corresponsabilidad. El cuidado humanizado aparece atado al modelo manicomial, culminando en prácticas enfocadas en el uso de la medicación, acciones desarticuladas y sin participación del paciente en el tratamiento. La percepción de la humanización es de dificultad de atención a las personas en crisis psíquicas que inviabiliza la producción del cuidado integral. Conclusión: el estudio contribuye a la reflexión del cuidado de enfermería en salud mental donde, hay que modificar las relaciones que el discurso biomédico mantiene con los que buscan una práctica humanizada.

Descriptores: Enfermería Psiquiátrica; Atención de Enfermería; Salud Mental; Humanización de la Atención. 


\section{Introdução}

A Política Nacional de Humanização (PNH) tem como premissa o reconhecimento dos usuários, trabalhadores e gestores em diferentes situações e contextos, apostando em novas práticas, relações e produção de conhecimentos orientados pelos princípios do Sistema Único de Saúde (SUS). Esta política aponta mudanças na produção de saúde, gestão e no cuidado, com ênfase no trabalho coletivo, na educação permanente dos profissionais de saúde e na formação de acadêmicos desta área de trabalho(1-2).

Apesar dos avanços na proposta da $\mathrm{PNH}$, ainda é frequente a fragmentação do processo de trabalho e das relações, a sobreposição do saber científico e biomédico ao senso comum, a separação do corpo e mente na prática de atenção e a desvalorização dos direitos dos usuários e trabalhadores. São desafios para o desenvolvimento de práticas de saúde, de caráter ampliado e colaborativo, que considerem o sujeito em seu contexto social(3-4).

No que tange a respeito da saúde mental, a humanização ganhou mais ênfase com o movimento da Reforma Psiquiátrica no Brasil, quando aconteceram a construção de espaços substitutivos de atenção diferenciada do modelo manicomial e a busca pela superação social e cultural dos estigmas vinculados à loucura(5-6).

A articulação entre a PNH e a Política de Atenção à Saúde Mental é capaz de possibilitar um modelo de atenção aberto e comunitário, que ofereça um cuidado integral ao usuário. Diante disso, o acolhimento, a autonomia, a corresponsabilidade e o protagonismo dos sujeitos são princípios da PNH considerados primordiais no cuidado em saúde mental por serem meios de transformação e qualificação das práticas e saberes em saúde ${ }^{(6-7)}$.

No âmbito da assistência em saúde mental, o agravamento do sofrimento psíquico, o predomínio do uso de terapias medicamentosas e as internações psiquiátricas recorrentes colocam os enfermeiros frente à necessidade de desenvolver ações que considerem a intersetorialidade, a interdisciplinaridade, o empoderamento dos usuários, além da aproximação da família e comunidade, colocando a humanização como um elemento estruturante do seu saber/fazer( ${ }^{(8)}$.

Desse modo, este estudo teve como objetivo conhecer as compreensões dos enfermeiros sobre humanização no cuidado em saúde mental.

\section{Método}

Trata-se de uma pesquisa com enfoque exploratório, de abordagem qualitativa, realizado em um hospital psiquiátrico do interior do Nordeste, Brasil. A instituição de saúde foi escolhida por fazer parte da organização dos serviços de saúde mental estabelecida pela gestão municipal e por ser referência nas regiões Oeste e Alto Oeste do Rio Grande do Norte para atuação frente às crises psíquicas, conforme aprovação pelos critérios do Programa Nacional de Avaliação dos Serviços Hospitalares - PNASH/Psiquiatria.

O referido local possui uma área superior a dez mil metros quadrados e está dividido em quatro unidades, sendo elas: a Unidade de Cuidados Femininos (UCF); a Unidade de Cuidados Masculinos (UCM); a Unidade de Cuidados Intensivos Femininos (UCIF) e ainda a Unidade de Cuidados Intensivos Masculinos (UCIM).

A população desta pesquisa foi composta por 17 enfermeiros que trabalhavam no hospital psiquiátrico. Os critérios de inclusão foram: ser enfermeiro atuante no estabelecimento há mais de seis meses e trabalhar diretamente com os pacientes. Destaca-se a exclusão dos enfermeiros que estavam de férias, com atestado médico ou materno e ainda que trabalhassem somente em turno noturno ou nos finais de semana. Assim, trabalhou-se com uma amostra final de 12 enfermeiros, levando em consideração uma amostragem por conveniência.

A entrada no campo foi preparada, previamente, pelo encontro com a coordenação de Enfermagem da referida instituição de saúde. Este contato foi iniciado por meio da entrega de um documento formal, que descrevia a solicitação do espaço hospitalar para a pesquisa, bem como os dias, os setores e os horários de observação presencial dos pesquisadores e a concordância em disponibilizar os registros de Enfermagem para a utilização de dados que enobrecessem o estudo. Ainda neste documento, foi descrita a metodologia a ser desenvolvida no local, reiterando o caráter ético do trabalho. Foi solicitado que as informações contidas no documento fossem compartilhadas com os enfermeiros do hospital de modo que todas as partes interessadas tomassem ciência do teor da pesquisa e da presença da pesquisadora no serviço.

A entrada em campo aconteceu em setembro de 2014, com duração de seis meses, o que permitiu contemplar os turnos matutinos e vespertinos, de segunda a sexta, das atividades realizadas pela Enfermagem. Cada enfermeiro, que permitiu a observação de suas atividades, formalizou sua participação na pesquisa com a assinatura do Termo de Consentimento Livre e Esclarecido (TCLE).

Para a coleta de dados do estudo, foram utilizadas a entrevista semiestruturada, a observação não participante e a observação dos registros de Enfermagem. Estas foram dirigidas e sistematizadas com base em um roteiro de observação do cuidado do enfermeiro no hospital psiquiátrico, levando em consideração os princípios da Política Nacional de Humanização: acolhimento, autonomia, protagonismo e corresponsabilidade.

As observações foram registradas em um diário de campo, que possibilitou uma maior aproximação com os 
sujeitos e o local de pesquisa, tornando os registros fonte de construção do conhecimento e reflexão da prática.

Em seguida, as entrevistas e os registros das observações foram analisados a partir da técnica de Análise de Conteúdo de Bardin dividida em três etapas básicas: pré-análise, na qual foram organizados os diários de campo a partir do roteiro de observação; na exploração do material, foram aplicadas técnicas específicas segundo o objetivo da pesquisa e na terceira etapa aconteceu o tratamento dos resultados e interpretações ${ }^{(9)}$.

Com o intuito de manter a preservação da identidade dos enfermeiros e a organização das observações e registros no diário de campo, foi utilizada a letra " $E$ " de enfermeiro seguida de algarismo arábico para identificá-los.

Sob a égide da Resolução 466/2012, esta pesquisa foi devidamente submetida ao Comitê de Ética e Pesquisa (CEP), sendo aprovada com parecer no CAAE 14628813.1.0000.5294.

\section{Resultados e Discussão}

Os participantes foram 12 enfermeiros de ambos os sexos, dez do sexo feminino e dois do sexo masculino, com idade média de 38 anos, entre os 26 e 58 anos. Eles trabalhavam no hospital psiquiátrico com período de um ano a 30 anos e média de tempo de serviço de dez anos e nove meses. Quanto à formação, dois possuíam graduação e dez, especialização, sendo destes seis na área de saúde mental.

As falas dos entrevistados foram evidenciadas e organizadas em unidades de registro, de acordo com suas semelhanças e pertinência, emergindo em um quantitativo de 82 unidades de análise de conteúdo agrupadas em quatro temas, que serão discutidos em quatro categorias: acolhimento, autonomia, protagonismo e corresponsabilidade.

\section{Acolhimento}

Observou-se que o acolhimento realizado pelos enfermeiros se destinava ao atendimento inicial do paciente no hospital psiquiátrico. Em uma sala pequena, intitulada de acolhimento, o enfermeiro realizava uma coleta de informações com o paciente que demonstrava cooperação; em outras situações, o atendimento era focado apenas na observação do seu estado físico ou nos relatos dos familiares.

A admissão dos pacientes acontece aqui no acolhimento. Eu preencho um checklist se o paciente falar, mas geralmente ele não fala ou está bem agressivo, então, pergunto à família. Tem família que exagera o quadro clínico do paciente, nesse caso, uso apenas a observação de como o paciente chega ao hospital para preencher o documento. (E2)
O enfermeiro visa mais à demanda de quem trouxe o paciente para o serviço de saúde do que o quê o paciente tem a dizer de sua angústia. Vale salientar que acolhimento é uma tecnologia do encontro, construído a cada dia, como espaço subjetivo de construção de vínculo e ressignificação de sofrimento, indo além do atendimento inicial ou do espaço físico do serviço de saúde. Por meio da escuta da história de vida, o acolhimento afirma relações de potência entre profissionais e usuários nos processos de produção de saúde ${ }^{(10-11)}$.

Na sala do acolhimento, é usado um checklist sobre o estado corporal do paciente constituído de perguntas rápidas, algumas de múltipla escolha.

Pergunto sobre a quantidade de internamentos anteriores; psiquiatra que acompanha o paciente; psicotrópicos que faz uso; queixa principal que desencadeou aquela crise; período em que foram identificadas as primeiras mudanças de comportamento e ainda dados pessoais e socioeconômicos. Depois, encaminho para o médico determinar o diagnóstico e o esquema medicamentoso. (E7)

Uma vez internado, o paciente é levado até sua ala e passa a viver situações diferentes do que estava acostumado. O enfermeiro do setor verifica o prontuário e registra sua admissão: o paciente chegou à UCIM em maca acompanhado pelo enfermeiro do acolhimento. Faz uso de hipertensivos, não verbaliza e possui ferimentos em várias partes do corpo. Terceiro internamento nesta instituição devido uso abusivo de álcool. Fica sob os cuidados da Enfermagem. (E9)

Essa forma de acolhimento demonstra seu formato nos moldes da psiquiatria tradicional, que se fundamenta apenas no referencial clínico centrado no adoecimento, na queixa orgânica e no diagnóstico médico. As observações demonstraram uma superficialidade das relações entre enfermeiros e pacientes em consequência da desvalorização da expressão dos sentimentos do paciente em sofrimento psíquico e dos acontecimentos de sua vida. Tal fato lança a necessidade de um acolhimento contínuo e em rede, desde a entrada até a saída do internamento, concretizando-o como um dos dispositivos essenciais no trabalho em saúde mental ${ }^{(10,12-13)}$.

\section{Autonomia}

Para adentrar a UCM, é preciso tocar a campainha da porta de acesso e aguardar que alguém a abra, pois o controle com as portas é bem rígido em todos os setores do hospital psiquiátrico. A porta foi aberta por um dos pacientes, que foi autorizado pela Enfermagem.

Alguns pacientes nós damos essa autonomia, mas não posso fazer isso com todos porque são muitos pacientes e temos que ficar sempre vigilantes. Hoje, eu tenho aqui mais de 50 pessoas só nesse setor. É muita gente para coordenar e a maioria não dá pra confiar. Claro que a autonomia deles é em decidir em que 
quarto vai ficar, a roupa que quer vestir, se quer se alimentar ou não, eles fazem trocas de produtos de higiene e chinelos. (E11)

A autonomia é considerada pelo enfermeiro como incentivo aos pacientes realizarem escolhas e algumas funções, reconhecendo os limites e as potencialidades do seu processo de adoecimento(14).

Eles querem mudar de quarto sempre, mas quem decide o seu quarto somos nós. Precisamos ficar atentos a tudo que acontece aqui. (E2)

Sua alta você já sabe, só vai acontecer quando você estiver bem mesmo, e é o médico que faz. (E4)

A relação de cuidado é permeada pela dificuldade do enfermeiro em ajudar os pacientes em sofrimento psíquico a realizarem suas escolhas de forma independente, uma vez que há maior necessidade de vigilância, em prol da manutenção do controle. Essas atitudes contribuem para o comprometimento da autonomia do paciente e fortalecimento da dependência à instituição

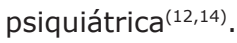

Cabe à instituição psiquiátrica, considerando o paciente em sofrimento psíquico como ser social e cidadão, funcionar como espaço de passagem à proporção em que possibilitaria, aos seus usuários, a autonomia em relação à continuidade do seu tratamento na rede de atenção psicossocial. Nesta perspectiva, surgem a necessidade de articulação com a rede de apoio e a busca de recursos no território. Já a Enfermagem precisa pensar na história de vida do paciente e em suas necessidades, desenvolvendo suas atividades a partir de uma escuta interessada na responsabilidade pelo cuidado, na relação de ajuda e na conquista da autonomia(15-16).

\section{Protagonismo}

Observou-se que o hospital psiquiátrico é um ambiente que dispensa o protagonismo do paciente. Ainda se cultiva sua hegemonia sobre as ações de saúde, reproduzindo um modelo de gestão pouco democrático, que não favorece a emergência de sujeitos transformadores dos modos de gerenciar e fazer saúde. Entretanto, o ser humano é um ser dependente de suas redes e, deste modo, as relações que envolvem os sujeitos impactam a construção de uma responsabilização compartilhada e protagonista(15).

Semana passada, eu estava fazendo uma anotação no prontuário e levei um murro do paciente. No outro dia, ela me pediu desculpa, disse que estava fora de si. Como vou pedir para uma pessoa como essa participar da construção de seu projeto terapêutico? Eu falo logo para o médico reavaliar suas medicações. (E5)

Para a construção de um projeto terapêutico, é necessário valorizar os saberes também do paciente no sentido de que ele tenha compromisso e protagonismo nas decisões, como um acordo com o profissional, evitando divisão, disputa e imposições. O protagonismo está ligado ao processo de construção de parcerias entre profissionais e pacientes à medida que todos elaboram conjuntamente alternativas para a condução a um caminho que respeite os valores culturais dos diferentes coletivos ${ }^{(2,4)}$.

Os enfermeiros buscam parcerias com as usuárias como forma de participação no tratamento e construção de saberes, porém, existe a acreditação de que sua doença afeta a capacidade de agir e pensar.

As pacientes daqui [UCF] nós deixamos bem livres porque elas são calmas, mas nas UCIF tem que ficar de olho, não pode deixar sozinhas porque são como crianças, não entendem bem as coisas. A gente tenta estabelecer uma parceria para que uma ajude as outras, se sintam úteis e participem mais do seu tratamento, mas, na maioria das vezes, a gente que toma decisões por elas serem doentes mentais. (E12)

Essa percepção limita determinadas decisões referentes ao tratamento, transparecendo mais uma vez existir uma relação hierárquica de saberes entre pacientes e enfermeiros e reforçando o papel dos enfermeiros que atuam em hospitais psiquiátricos de supervisionar e controlar ${ }^{(12)}$.

Os usuários tornam-se protagonistas quando ganham voz ativa diante das relações terapêuticas que considerem suas histórias de vida. Ainda é necessário romper com o método epistêmico da psiquiatria, o conceito de doença mental como erro e desrazão, bem como os princípios do tratamento moral que ainda embasam as condutas adotadas nos serviços de saúde ${ }^{(15,17)}$.

Por que você não quer almoçar, mulher? Vai almoçar, sim. Depois, vai ficar com fome e tem que tomar a medicação. Lembre disso para o estômago não doer. Você não quer ir embora daqui? Vai demorar mais se você não comer. (E10)

Observou-se que a conduta do enfermeiro é influenciada pelo poder centralizador do hospital psiquiátrico, que impõe regras muitas vezes diferentes das rotinas diárias do paciente. Desse modo, a angústia é mascarada pela medicalização do sofrimento, que não considera as questões do sujeito e impede que este elabore um saber sobre aquilo que o faz sofrer ou sobre seu cuidado. A valorização das falas destes pacientes pode fornecer subsídios importantes para que as ações em saúde mental possam ser repensadas em prol de um cuidado integral e humanístico(18).

\section{Corresponsabilidade}

A corresponsabilidade implica o envolvimento dos profissionais de saúde, a participação do paciente nos planos e ações terapêuticos e a articulação entre os serviços de saúde e de apoio(1). No entanto, a percepção 
do enfermeiro sobre o cuidado humanizado em saúde mental é de encaminhamento, sinônimo de trabalho difícil.

Os médicos aqui ficam de sobreaviso. A gente liga e eles vêm aqui para mudar a medicação e reavaliar a prescrição. Não temos muito que fazer, se passar mal, ligo para o médico, se estiver em crise e quiser conversar, encaminho para o psicólogo. (E1)

Tem uns pacientes que são bem graves mentalmente e que possuem outras doenças também. Realizar um cuidado humanizado nestas condições que o hospital se encontra é muito difícil, imagine em uma situação de urgência, é mais difícil ainda. (E6)

Observou-se que os entrevistados negam o cuidado ao paciente em crise psíquica como sua responsabilidade e passam essa função a outros profissionais. Outro fato verificado foi a falta de instrumentos básicos, como pontos de oxigênio e desfibrilador, para um atendimento de urgência ou emergência no hospital psiquiátrico, sendo sempre necessário acionar o Serviço de Atendimento Móvel de Urgência (SAMU) para transporte do paciente a um hospital geral.

Essas situações demonstram a necessidade de construção de novas relações e práticas de corresponsabilidade com vistas a atender às necessidades de saúde dos pacientes. Vale ressaltar que o reconhecimento, por parte dos enfermeiros e demais profissionais, dos elementos assistenciais, subjetivos e sociais, é primordial para o um cuidado humanizado ${ }^{(5,19)}$.

Os enfermeiros relataram também a dificuldade de sensibilizar o paciente para ser corresponsável pelo seu tratamento, bem como de envolver outros enfermeiros e de conscientizar a família.

É difícil chamar essa responsabilidade do tratamento para os pacientes porque muitos nem querem. A gente se sente sozinho porque os outros colegas enfermeiros também cansam. A gente sabe que precisa cuidar com humanização, mas é difícil. O paciente já se acostumou em só tomar o remédio e a família apoia e acha ruim quando não sai daqui com mais um medicamento na lista. (E8)

Este contexto reitera o trabalho fragmentado, que pode maximizar o sofrimento em que o paciente já se encontra, desestimulando sua capacidade de enfrentamento diante de seus problemas e seu tratamento.

O ambiente asilar e a supressão dos sintomas, inclusive com a medicalização do sofrimento, evidenciam a falta de um aprofundamento em ferramentas clínicas que rompam com essa posição de objetificação daquele que sofre e com essa imposição de condutas corretivas a partir de suas próprias inquietações ${ }^{(10,16)}$.

A perspectiva de se dar voz ao sofrimento do paciente, baseada na ampliação da clínica e enfoque no sujeito, implica assumir uma responsabilidade na mudança no cuidado. Esta pode ser pensada em tecnologias em saúde que possibilitem compreender o sofrimento psíquico a partir do contexto do usuário, valorizando suas experiências e atentando para suas necessidades, incluindo o cuidado aos diferentes aspectos que compõem o cotidiano desse indivíduo(20).

\section{Conclusão}

Os resultados encontrados demonstraram que o cuidado de Enfermagem em saúde mental, considerando os princípios da humanização, não se efetiva nas relações entre enfermeiros, pacientes e outros profissionais.

O acolhimento, a autonomia, o protagonismo e a corresponsabilidade estiveram atrelados ao modelo manicomial, culminando em práticas mais focadas no uso da medicação, ações desarticuladas e sem participação do paciente no tratamento. A percepção da humanização que permeia os enfermeiros é de dificuldade de atenção às pessoas em crises psíquicas, o que inviabiliza a produção de um cuidado integral.

Salienta-se que a coleta de dados aconteceu em um único serviço de saúde mental, o que pode ser considerado uma limitação da pesquisa. Para o desenvolvimento de futuros estudos, deve-se aprofundar a discussão de estratégias que materializem a incorporação dos princípios da humanização para os enfermeiros e na relação com usuários.

Este estudo contribui para que a Enfermagem avalie suas práticas e relações, considerando os ideais da Reforma Psiquiátrica Brasileira, haja vista que não basta destruir os muros do hospício, é preciso modificar as relações que o discurso biomédico mantém com os que buscam sua prática, além de mudar o saber psiquiátrico tradicional impregnado nas instituições e práticas.

\section{Referências}

1. Ministério da Saúde (BR). Secretaria de Atenção à Saúde. Núcleo Técnico da Política Nacional de Humanização. HumanizaSUS: Documento base para gestores e trabalhadores do SUS. Brasília: Secretaria de Atenção à Saúde, Núcleo Técnico da Política Nacional de Humanização; 2010.

2. Ministério da Saúde (BR). Cadernos HumanizaSUS: formação e intervenção. Brasília (DF): Ministério da Saúde; 2010. (Série B. Textos Básicos de Saúde).

3. Morschel A, Barros MEB. Processos de trabalho na saúde pública: Humanização e efetivação do Sistema Único de Saúde. Saúde Soc. [Internet]. 2014;23(3):928-41. [Acesso 27 nov 2017]. Disponível em: http://www. scielo.br/scielo.php?script =sci_arttext\&pid=S010412902014000300928\&lng=en. http://dx.doi.org/10.1590/ s0104-12902014000300016. 
4. Martins CP, Luzio CA. Política HumanizaSUS: ancorar uma nave no espaço. Interface. (Botucatu). [Internet]. 2017; 21(60):13-22. [Acesso 2 dez 2017]. Disponível em: http://www.scielo.br/scielo.php?script $=$ sci_ arttext\&pid=S1414-32832017000100013\&lng=en. http:// dx.doi.org/10.1590/1807-57622015.0614.

5. Chernicharo IM, Silva FD, Ferreira MA. Description of the term humanization in care by nursing professionals. Esc Anna Nery. [Internet]. 2014;18(1):156-62. [Acesso 2 dez 2017]. Disponível em: http://www. scielo.br/scielo.php?script=sci_arttext\&pid=S1414$81452014000100156 \& / n g=e n$. http://dx.doi. org/10.5935/1414-8145.20140023.

6. Santos ST, Lima FAC. Interfaces da política nacional de humanização de assistência à saúde de pessoas com transtorno mental. Rev Iberoam Educ Invest Enferm. [Internet]. 2017; 7(3):65-77. [Acesso 10 dez 2017]. Disponível em: https://www.enfermeria21.com/revistas/ aladefe/articulo/261/interfaces-da-politica-nacional-dehumanizacao-de-assistencia-a-saude-de-pessoas-comtranstorno-mental/.

7. Branco $A B A C$, Bosi MLM. Integralidade e humanização em saúde mental: experiências de usuários em dois centros de atenção psicossocial. Cad Saúde Coletiva. [Internet]. 2014;22(2):221-2. [Acesso 16 dez 2017]. Disponível em: http://www.scielo.br/scielo.php?script $=$ sci_ arttext\&pid=S1414-462X2014000200221\&lng=en. http:// dx.doi.org/10.1590/1414-462X201400020018.

8. Oliveira LC, Silva RAR, Medeiros MN, Queiroz JC, Guimarâes J. Humanized care: discovering the possibilities in the practice of nursing in mental health. J Res Fundam Care. online. [Internet]. 2015;7(1):1774-82. [cited Dec 16 2017]. Available from: http://www.seer.unirio.br/index. php/cuidadofundamental/article/view/3396/pdf_1402.

9. Bardin L. Análise de conteúdo. $5^{a}$ ed. Lisboa: Edições 70; 2011. 229 p.

10. Lima DWC, Silveira LC, Vieira AN, Cunha BMC, Almeida ANS, Guerreiro EM. Theoretical references that guide nursing practice in mental health. Esc Anna Nery. [Internet]. 2014;18(2):336-42. [cited Jan 12 2018]. Available from: http://www.scielo.br/scielo.php?script=sci_ arttext\&pid=S1414-81452014000200336\&lng=en. http:// dx.doi.org/10.5935/1414-8145.20140049.

11. Maynart WHDC, Albuquerque MCDS, Brêda MZ, Jorge JS. A escuta qualificada e o acolhimento na atenção psicossocial. Acta Paul Enferm. [Internet]. 2014;27(4):300-3. [Acesso 18 jan 2018]. Disponível em: http://www.scielo.br/scielo.php?script $=$ sci_ arttext\&pid=S0103-21002014000400003\&lng=en. http:// dx.doi.org/10.1590/1982-0194201400051.

12. Baptista MKS, Santos RM, Duarte SJH, Comassetto I, Trezza MCSF. The patient and the relation between power-knowledge and care by nursing professionals. Esc Anna Nery. [Internet]. 2017;21(4):1-9. [cited
Jan 20 2018]. Available from: http://www.scielo. br/scielo.php?script $=$ sci_arttext\&pid=S1414$81452017000400205 \& / n g=e n$. http://dx.doi. org/10.1590/2177-9465-ean-2017-0064.

13. Pegoraro RF, Bastos LSN. Experiências de acolhimento segundo profissionais de um centro de atenção psicossocial. Rev Enferm Atenção Saúde Online. [Internet]. 2017;6(1):3-17. [Acesso 18 jan 2018]. Disponível em: http://seer.uftm.edu.br/revistaeletronica/index.php/enfer/ article/view/1525/pdf.

14. Dutra VFD, Bossato HR, Oliveira RMP. Mediar a autonomia: um cuidado essencial em saúde mental. Esc Anna Nery. [Internet]. 2017;21(3):e20160284. [Acesso 2 dez 2017]. Disponível em: http://www. scielo.br/scielo.php?script $=$ sci_arttext\&pid=S1414$81452017000300205 \&$ Ing $=$ pt. http://dx.doi. org/10.1590/2177-9465-ean-2016-0284.

15. Yasui S, Luzio CA, Amarante P. From manicomial logic to territorial logic: Impasses and challenges of psychosocial care. J Health Psychol. [Internet]. 2016;21(3):400-8. [cited Jan 23 2018]. Available from: doi: $10.1177 / 1359105316628754$.

16. Almeida ANS, Feitosa RMM, Boesmans EF, Silveira LC. Cuidado clínico de enfermagem em saúde mental: reflexões sobre a prática do enfermeiro. J Res Fundam Care online. [Internet]. 2014; 6(1):213-34. [Acesso 23 jan 2018]. Disponível em: https://www.redalyc.org/ articulo. $0 a$ ? id $=505750621016$.

17. Costa DFC, Paulon SM. Social participation and protagonism in mental health: the rising of a collective. Saúde Debate. [Internet]. 2012;36(95):572-82. [cited Fev 03 2018]. Available from: http://www. scielo.br/scielo.php?script $=$ sci_arttext\&pid=S010311042012000400009\&Ing=en. http://dx.doi.org/10.1590/ S0103-11042012000400009.

18. Lima DWC, Silveira LC, Vieira AN. A escuta terapêutica no cuidado clínico de enfermagem em saúde mental. Texto Contexto Enferm. [Internet]. 2015;24(1):154-60. [Acesso 23 fev 2018]. Disponível em: http://www.scielo.br/pdf/ tce/v24n1/pt_0104-0707-tce-24-01-00154.pdf.

19. Lima MS, Aguiar ACL, Sousa MMS. O cuidado compartilhado em saúde mental como potencial de autonomia do usuário. Psicol Estud. [Internet]. 2015;20(4):675-86. [Acesso 3 fev 2018]. Disponível em: https://www.redalyc.org/pdf/2871/287145780015.pdf. 20. Abreu TFK, Amendola F, Trovo MM. Tecnologias relacionais como instrumentos para o cuidado na Estratégia Saúde da Família. Rev Bras Enferm. [Internet]. 2017;70(5):981-7. [Acesso 3 fev 2018]. Disponível em: http://www.scielo.br/scielo.php?script $=$ sci_ arttext\&pid=S0034-71672017000500981\&lng=pt. http:// dx.doi.org/10.1590/0034-7167-2016-0337 


\section{Contribuição dos Autores}

Concepção e planejamento do estudo: Deivson Wendell da Costa Lima. Obtenção dos dados: Deivson Wendell da Costa Lima, Ana Karla Ramalho Paixão. Análise e interpretação dos dados: Deivson Wendell da Costa Lima, Ana Karla Ramalho Paixão. Redação do manuscrito: Deivson Wendell da Costa Lima, Ana Karla Ramalho Paixão, Lívia Dayane Sousa Azevedo. Revisão crítica do manuscrito: Deivson Wendell da Costa Lima, Kelianny Pinheiro Bezerra, Rodrigo Jácob Moreira de Freitas, Fátima Raquel Rosado Morais.

Todos os autores aprovaram a versão final do texto.

Conflito de interesse: os autores declararam que não há conflito de interesse.

Copyright @ 2021 SMAD, Rev. Eletrônica Saúde Mental Álcool Drog. Este é um artigo de acesso aberto distribuído sob os termos da Licença Creative Commons CC BY-NC.

Esta licenca permite que outros remixem, adaptem e criem a partir do seu trabalho para fins não comerciais, e embora os novos trabalhos tenham de lhe atribuir o devido crédito e não possam ser usados para fins comerciais, os usuários não têm de licenciar esses trabalhos derivados sob os mesmos termos. 